\section{Consumo de mono, di, oligo sacáridos y polioles fermentables (FODMAPs), una nueva fuente de sintomatología gastrointestinal}

\author{
CATALINA GREZ $^{1, \mathrm{a}}$, ÁLVARO VEGA ${ }^{1, \mathrm{a}}$, MAGDALENA ARAYA ${ }^{1}$
}

\section{Fermentable oligosaccharides, disaccharides, monosaccharides and polyols (FODMAPS)}

An increasing number of children and adults are currently suffering symptoms due to FODMAP (Fermentable Oligosaccharides, Disaccharides, Monosaccharides and Polyols) consumption. These carbohydrates are poorly digested in the human gastrointestinal tract, exerting an osmotic effect in the small intestine. In the colon, they become substrates to the microbiota. Microbial fermentation explains symptoms such as abdominal distention (postprandial fullness), bloating and flatulence, abdominal pain and loose feces or diarrhea. There are no standardized methods to measure them in daily clinical work. Daily tolerance and the no-effect doses are unclear. How to diagnose and treat FODMAP associated symptoms is also controversial. In this review, we aim to define FODMAP, their associated symptoms and the current techniques for assessing them. The low-FODMAP diet is described and how to implement it.

(Rev Med Chile 2019; 147: 1167-1175)

Key words: Abdominal Pain; Fermentation; Microbiota.
'Instituto de Nutrición y

Tecnología de los Alimentos (INTA), Universidad de Chile.

Santiago, Chile.

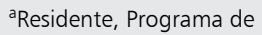
Gastroenterología Infantil, Facultad de Medicina, Universidad de Chile. Santiago, Chile

Trabajo no recibió financiamiento. Los autores declaran no tener conflicto de interés.

Recibido el 25 de enero de 2019, aceptado el 12 de junio de 2019 .

Correspondencia a: Dra. Magdalena Araya Instituto de Nutrición y Tecnología de los Alimentos. Santiago, Chile. maraya@inta.uchile.cl
$\mathrm{H}$ oy, un sector creciente de niños y adultos desarrollan síntomas debidos al consumo de FODMAPs (mono, di, oligo sacáridos y polioles fermentables, en su sigla en inglés), carbohidratos que se digieren pobremente en el sistema gastrointestinal humano y son fermentados por la microbiota colónica. Más que una enfermedad propiamente tal, el aumento de sintomatología hoy día se relaciona con "comer mucho", tanto alimentos como productos procesados. La evidencia acerca de estos efectos se basa principalmente en las propiedades de los FODMAPs (ver abajo), ya que los ensayos en seres humanos son escasos. No hay métodos estandarizados acerca de cómo medir la tolerancia, el consumo diario que se asocia a No-efecto, cómo diagnosticarlos ni cómo tratarlos. Esto hace que hoy día constituyan un tema de interés, se investiguen activamente y esto nos motivó a realizar esta revisión, que por las razones expuestas, no es definitiva. El objetivo es definir qué son los FODMAPs, plantear el problema que provoca su alto consumo y aportar conceptos relevantes para su manejo en la clínica diaria.

\section{Historia}

Históricamente, la aparición de síntomas gastrointestinales molestos se asoció con alimentos como leche, legumbres, algunas verduras, frutas y granos ${ }^{1}$. Hoy se conocen los componentes de los alimentos responsables de generar estos síntomas. En 1965 se describió la intolerancia a la lactosa ${ }^{2}$; en 1976 el concepto se amplió a productos usados como ingredientes de alimentos procesados, describiéndose que el xilitol (endulzante poliol) 
puede producir diarrea ${ }^{3}$. En 1978 se reportaron casos de diarrea crónica manejados con dieta libre de fructosa ${ }^{4}$ y en 1987 se comunicó que el consumo de oligosacáridos usados como endulzantes, generaba meteorismo ${ }^{5}$. En 2005, se utilizó por primera vez un término que agrupaba a los carbohidratos de cadena corta y polioles pobremente absorbidos $\mathrm{y}$ altamente fermentables: FODMAPs ${ }^{6}$.

\section{Definiciones}

Monosacárido: Fructosa. Presente en la dieta como monosacárido libre, como constituyente del disacárido sacarosa o polimerizada como fructanos. Cuando la fructosa es ingerida como sacarosa en combinación equimolar con glucosa, la capacidad de absorción es alta. La fructosa en exceso de glucosa también es absorbida, pero mediante un mecanismo de baja capacidad, lo que se manifiesta como malabsorción ${ }^{7}$.

Disacárido: Lactosa. Disacárido de la leche formado por una galactosa y una glucosa. Su malabsorción hace que llegue al colon donde es fermentada por la microbiota. La deficiencia de la enzima lactasa tiene alta prevalencia en la población, aunque con importantes variaciones entre grupo étnicos y países ${ }^{8}$.

Oligosacáridos: Fructanos y Galactooligosacáridos. Los fructanos corresponden a oligosacáridos de fructosa sintetizados a partir de sacarosa, que presentan una glucosa en uno de sus extremos. Se denominan fructooligosacáridos (FOS) a los fructanos con menos de 10 unidades de fructosa e inulinas aquellos con más de 10 unidades; sin embargo, la denominación de inulinas deriva del tipo de enlace entre los monómeros de fructosa y no del número de éstas, lo que suele llevar a confusión?. Los galactooligosacáridos (GOS) son sintetizados a partir de sacarosa, compuestos por unidades variables de galactosa unidas a glucosa y fructosa en un extremo. La rafinosa y estaquiosa representan la mayoría de los GOS de la dieta, ya sea contenidos naturalmente en legumbres y derivados de la soya o porque son utilizados como prebióticos ${ }^{10}$.

Polioles. También denominados polialcoholes o azúcares alcohólicos, son alcoholes derivados de monosacáridos que han sido hidrogenados. Los más frecuentes en los alimentos son el sorbitol (derivado de la glucosa) y el manitol (derivado de la manosa). Otros polioles son lactitol, xilitol, maltitol y eritritol. Están presentes naturalmente en frutas y además son usados como endulzantes, principalmente en chicles y caramelos sin azúcar. Aparentemente son absorbidos en forma lenta vía difusión pasiva, lo que explicaría por qué generan síntomas ${ }^{11}$.

\section{Mecanismos de acción}

El sistema enzimático que procesa FODMAPs en el tracto digestivo humano es limitado para manejar cada ingesta (Tabla 1). Así, permanecen en el lumen intestinal ejerciendo efecto osmótico, que es mayor cuando se trata de moléculas pequeñas como monosacáridos y polioles. El aumento de agua en el lumen promueve la peristalsis, avanzan rápidamente al colon y son fermentados por la microbiota que los utiliza como sustrato ${ }^{12}$. Estos fenómenos se han demostrado in vivo mediante resonancia magnética, que permitió estimar el volumen de agua en el intestino delgado y la distensión colónica. Cuando voluntarios sanos ingirieron una solución de manitol o glucosa, a igual concentración, se observó que después de una hora el aumento del volumen intestinal producido por manitol era 6 veces mayor que el producido por glucosa ${ }^{13}$. Igualmente, tras $75 \mathrm{~min}$ de ingerir la misma cantidad de glucosa, fructosa o inulina, la fructosa produjo un aumento del volumen intestinal mayor que los otros compuestos, que disminuyó cuando estaba combinado con glucosa, y que tanto fructosa como inulinas producían mayor distensión colónica ${ }^{14}$. Evaluando la asociación entre ingesta y aparición de síntomas, se observó que en pacientes con síndrome de intestino irritable (SII) que ingirieron inulinas, los síntomas gastrointestinales desarrollados eran concordantes con el aumento de gas en el colon ${ }^{15}$.

\section{Cuantificación de FODMAPS en los alimentos}

Los alimentos que contienen FODMAPs son numerosos, incluyendo tanto vegetales como alimentos procesados (Tabla 2). El grupo de la Universidad de Monash cuantifica FODMAPs utilizando un protocolo consistente en adquirir alimentos en el comercio, separar la porción comestible o cocinarlos en caso de ser necesario, 
Tabla 1. Vía de absorción y efectos gastrointestinales de los principales subgrupos de FODMAPs

\begin{tabular}{|c|c|c|c|}
\hline FODMAP & Subgrupo & Absorción en intestino delgado & Efecto en el tracto gastrointestinal \\
\hline Monosacáridos & Fructosa & $\begin{array}{l}\text { Difusión facilitada vía transportador } \\
\text { GLUT5 (baja capacidad) } \\
\text { Transporte activo con glucosa vía } \\
\text { transportador GLUT2 (alta capacidad) }\end{array}$ & $\begin{array}{l}\text { Aumento de contenido de agua intestinal } \\
\text { por efecto osmótico con o sin malabsorción. } \\
\text { Aumento de gas en colon por fermentación } \\
\text { de proporción no absorbida }\end{array}$ \\
\hline Disacáridos & Lactosa & $\begin{array}{l}\text { Requiere digestión por lactasa } \\
\text { Malabsorción en personas con déficit } \\
\text { de lactasa }\end{array}$ & $\begin{array}{l}\text { Aumento de contenido de agua intestinal } \\
\text { por efecto osmótico de lactosa no digerida. } \\
\text { Aumento de gas en colon por fermentación } \\
\text { colónica }\end{array}$ \\
\hline Oligosacáridos & $\begin{array}{l}\text { Fructanos } \\
\text { (FOS e inulinas) } \\
\text { GOS }\end{array}$ & $\begin{array}{l}\text { Sin absorción por ausencia de hidrola- } \\
\text { sa intestinal }\end{array}$ & $\begin{array}{l}\text { Aumento de gas en colon por fermentación } \\
\text { colónica }\end{array}$ \\
\hline Polioles & $\begin{array}{l}\text { Sorbitol } \\
\text { Manitol } \\
\text { Xilitol } \\
\text { Maltitol } \\
\text { Eritritol } \\
\text { Isomaltitol }\end{array}$ & $\begin{array}{l}\text { Difusión pasiva lenta a lo largo del } \\
\text { intestino delgado }\end{array}$ & $\begin{array}{l}\text { Aumento de contenido de agua intestinal } \\
\text { por efecto osmótico con o sin malabsorción. } \\
\text { Aumento de gas en colon por fermentación } \\
\text { de proporción no absorbida }\end{array}$ \\
\hline
\end{tabular}

FOS: Fructooligosacáridos; GOS: Galactooligosacáridos.

Tabla 2. Contenido de los principales FODMAPs (fructosa, lactosa, fructanos y galacto-oligosacáridos y polioles) en alimentos de uso habitual

\begin{tabular}{|c|c|c|c|c|}
\hline & Frutas & Vegetales & Edulcorantes & Alcohol \\
\hline Fructosa & $\begin{array}{l}\text { Manzana, mango, } \\
\text { pera, sandia, chirimo- } \\
\text { ya, higos, guindas. } \\
\text { Jugos de frutas }\end{array}$ & $\begin{array}{l}\text { Tomates secos, } \\
\text { esparragos, alcachofa, } \\
\text { arvejas }\end{array}$ & $\begin{array}{l}\text { Miel, jarabe de maíz } \\
\text { alto en fructosa }\end{array}$ & Ron, vinos dulces \\
\hline Lactosa & $\begin{array}{l}\text { Quesos: ricota, moza- } \\
\text { rela, cottage, queso } \\
\text { fresco }\end{array}$ & $\begin{array}{l}\text { Leche: cabra, vaca, } \\
\text { oveja, bufalo }\end{array}$ & $\begin{array}{l}\text { Otros lacteos: yogurt, } \\
\text { helado, leche con- } \\
\text { densada, evaporada, } \\
\text { crema }\end{array}$ & \\
\hline $\begin{array}{l}\text { Fructanos y Galacto- } \\
\text { oligosacáridos }\end{array}$ & $\begin{array}{l}\text { Frutas: pomelo, san- } \\
\text { dia, ciruela, durazno, } \\
\text { higos, platano, pasas, } \\
\text { melón verde, caqui }\end{array}$ & $\begin{array}{l}\text { Vegetales: ajo, cebolla, } \\
\text { cebollín, ajo en polvo, } \\
\text { alcachofa }\end{array}$ & $\begin{array}{l}\text { Frutos secos y granos: } \\
\text { trigo, centeno, } \\
\text { cebada, pistachos, } \\
\text { almendras, castañas, } \\
\text { mani }\end{array}$ & $\begin{array}{l}\text { Legumbres: garban- } \\
\text { zos, lentejas, porotos, } \\
\text { soja, habas }\end{array}$ \\
\hline Polioles & $\begin{array}{l}\text { Frutas: nectarines, } \\
\text { duraznos, mora, guin- } \\
\text { das, ciruelas, sandia, } \\
\text { damazco, palta, coco, } \\
\text { granada. Jugos de } \\
\text { frutas }\end{array}$ & $\begin{array}{l}\text { Vegetales: coliflor, } \\
\text { champiñones, zapallo } \\
\text { calabacín, apio }\end{array}$ & $\begin{array}{l}\text { Agentes endulzantes: } \\
\text { sorbitol, manitol, } \\
\text { xylitol, isomaltitol, } \\
\text { maltitol, lactitol }\end{array}$ & $\begin{array}{l}\text { Otros: caramelos } \\
\text { duros, masticables } \\
\text { y chicles sin azúcar, } \\
\text { mermeladas y conser- } \\
\text { vas, productos hor- } \\
\text { neados como queques } \\
\text { o pasteles. Wasabi }\end{array}$ \\
\hline
\end{tabular}

homogenizarlos y congelar alícuotas de $100 \mathrm{~g}$ que son desecadas en frío. Posteriormente, se mide la cantidad y tipo de FODMAP presente mediante cromatografía líquida o métodos enzimáticos ${ }^{16-18}$. Para definir un alimento como bajo o alto en FOD-
MAPs, este grupo ha propuesto valores de corte arbitrarios para cada uno, en base a la cantidad presente en una porción "habitual" de alimentos durante una comida, que produzca síntomas en personas con SII. Si la porción definida como 
habitual de un alimento contiene un FODMAP específico en mayor cantidad que el valor propuesto, se considera alto en FODMAPs. Con el objetivo de planificar una dieta baja en FODMAPs (ver más adelante), se proponen puntos de corte para el total de FODMAPs presentes en una comida ${ }^{19}$. Aunque no están estandarizados, estos valores han sido probados en algunos estudios clínicos con aparentes buenos resultados ${ }^{20}$, por lo que se siguen utilizando.

\section{Fisiopatología y sintomatología}

Los mecanismos fisiopatológicos que despiertan los FODMAPs son la base para entender la sintomatología que inducen (Tabla 1); también se acepta que podrían ejercer efectos directos mediante mecanismos poco conocidos $^{21}$. Los polisacáridos fermentados en el colon producen energía adicional y diversos metabolitos, como ácidos grasos de cadena corta, de conocida acción beneficiosa en la fisiología intestinal, por lo que se ha postulado que los polisacáridos fermentables tendrían una acción prebiótica benéfica para el huésped $^{22}$, especialmente en enfermedades metabólicas como obesidad, diabetes o resistencia a insulina ${ }^{23-25}$. El efecto final observado depende del contrapunto entre su acción como fibra fermentable y su mayor o menor capacidad de inducir efecto glicémico.

Las manifestaciones clínicas que afectan el tracto gastrointestinal (Figura 1) incluyen reflujo gastroesofágico, acidez, disminución del apetito, eructos, distensión abdominal/meteorismo, ruidos hidroaéreos, gases, dolor abdominal de tipo cólico, dolor epigástrico y cambio del hábito intestinal, desde deposiciones más blandas y frecuentes a diarrea. Se ha descrito cefalea, vértigo, alteraciones de la memoria y letargia en menos de $20 \%$ de pacientes con intolerancia a carbohidratos $^{26,27}$, y hoy se discute si los FODMAPs son capaces de producir manifestaciones extraintestinales. Se postula que estas últimas podrían deberse a la producción de metabolitos capaces de alterar la señalización celular, pero los mecanismos responsables de la aparición de uno u otro síntoma no están claros ${ }^{21}$. Sin duda la fermentación en el colon es responsable de buena parte de la sintomatología, pero existen numerosos factores contribuyentes, aportados tanto por el huésped como por los compuestos presentes en el lumen.

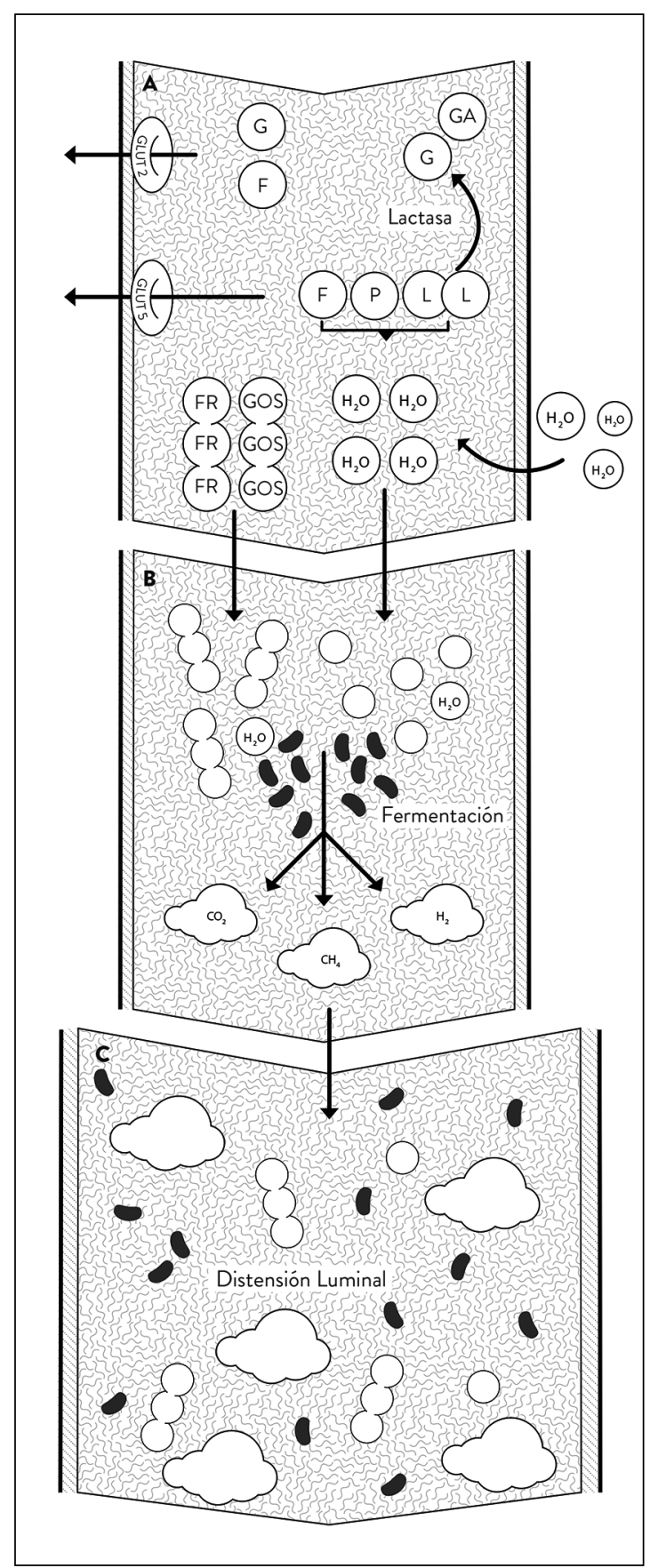

Figura 1. Mecanismos involucrados y efectos asociados al consumo de FODMAPs. A. Digestión de carbohidratos en el intestino delgado donde ocurre la hidrólisis de di y polisacáridos y la acumulación de agua debido al efecto osmótico que ejercen los FODMAPs. B. Llegada de los FODMAPs al intestino grueso donde la microbiota los fermenta. C. Aumento del contenido de agua y producción de gas que inducen los efectos observables. 


\section{Factores que aporta el huésped}

La motilidad intestinal, el tiempo de tránsito, la actividad enzimática presente y la composición de la microbiota intestinal influyen en la digestión, absorción, fermentación de los alimentos y los efectos observables. También participan otras características del individuo, algunas no modificables como el sexo, genética, personalidad, y otras que varían en el tiempo, como edad, peso, salud. El estilo de vida es relevante, ya que factores como el patrón de consumo de FODMAPs, uso de medicamentos y drogas y nivel de actividad física influyen el funcionamiento gastrointestinal. El tránsito oro-fecal tiene alta variabilidad individual ${ }^{28}$, es influenciado por la calidad de la dieta, y a su vez tiene capacidad de modificar la microbiota colónica ${ }^{29}$. Las enfermedades que sufra el individuo también influyen en los síntomas observados; por ejemplo, los pacientes celíacos tienen habitualmente cierto grado de deficiencia de lactasa y esto hará variar la cantidad de sustrato que se hará disponible a la microbiota del colon. En pacientes con enfermedad de Crohn, el contenido de proteínas, mucus y sangre en la mucosa inflamada promueven el efecto osmótico y la diarrea exudativa ${ }^{30}$.

\section{Factores aportados por los FODMAPs}

Las características locales del medio y la estructura química de los alimentos influyen en la posibilidad de aparición de efectos. Por ejemplo, la matriz sólida/líquida que porta el compuesto influye en la velocidad de tránsito; los líquidos son más rápidamente absorbidos y mejor tolerados ${ }^{31}$; el consumo durante una comida también aumenta la aceptabilidad ${ }^{32}$. La presencia de grasas y otros componentes capaces de enlentecer el tránsito intestinal disminuyen el efecto observable. La composición físico-química de los alimentos también cumple un rol modulador, por ejemplo, el número de unidades de azúcar y sus grupos funcionales, los enlaces químicos presentes, las ramificaciones en la molécula, el grado de polimerización y el tamaño molecular ${ }^{33}$. Por ejemplo, el sorbitol es una glucosa que por tener un grupo hidroxilo adicional se absorbe más lentamente. La sacarosa es completamente digerible, pero el isomaltitol, por tener uniones alfa 1-6, resulta más resistente a la digestión enzimática. Los FODMAPs de menor grado de polimerización tienen menor peso molecular y ejercen mayor efecto osmótico ${ }^{34}$.
Si los FODMAPs consumidos sobrepasan la capacidad de fermentación de la microbiota colónica, el contenido luminal, que ya tenía mayor cantidad de agua por el efecto osmótico ejercido en sectores altos del intestino, es excretado aumentando el gas y el volumen de deposición emitida $^{32,34}$. La cantidad total consumida afecta la tolerancia clínica aparente, por ejemplo, la presencia de varios compuestos con efecto laxante favorece la aparición de diarrea. El consumo de compuestos con efecto pre o probiótico, estimulan el crecimiento de bifidobacterias y lactobacilos, que ayudarán a la digestión de otros FODMAPs presentes $^{35}$. El efecto contrario se produce con la administración de antibióticos que reduzcan las especies capaces de fermentar los FODMAPs presentes en el lumen.

\section{FODMAPs e intestino irritable}

Buena parte de los estudios disponibles están hechos en pacientes con SII y los resultados no son concluyentes. Inicialmente, estudios de individuos con SII y sin enfermedad celíaca (EC), mostraron que la presencia de gluten en la dieta puede inducir síntomas, lo que sugiere que o bien el grupo SII incluye individuos con sensibilidad no celíaca al gluten/trigo (SNCG), o que quienes sufren SII pueden responder a los fructanos del trigo, y eso explicaría el efecto beneficioso de la dieta libre de gluten (DLG) ${ }^{36,37}$. En 72 pacientes mayores de 16 años con SII (Roma III) y sin EC, se administró DLG por 6 semanas y luego se desafió reintroduciendo gluten $(\mathrm{n}=35)$ o placebo $(\mathrm{n}=37)^{38} ; 83,8 \%$ y $25,7 \%$ respectivamente mejoraron la escala de síntomas en general $(\mathrm{p}<0,001)$, pero no la de síntomas específicos. Esto también se interpretó como que un subgrupo sufriría SNCG y responde al gluten. Comparando una dieta baja en FODMAPs con la dieta de tratamiento "habitual" (según criterios NICE), 92 pacientes adultos con SII mostraron significativamente mayor frecuencia de resolución de molestias que los que siguieron la dieta "habitual" 39 . En Suecia ${ }^{40}$, un estudio de 67 adultos con SII (Roma III) que siguieron una dieta pobre en FODMAPs $(\mathrm{n}=33)$ o la dieta "habitual" $(\mathrm{n}=34)$ por 4 semanas, mostraron que el score de sintomatología disminuyó en ambos grupos $(\mathrm{p}<0,0001)$, pero no hubo diferencias entre los dos tipos de tratamiento $(\mathrm{p}=0.72)$. En 12 niños 
con SII, una dieta baja en FODMAPs redujo la frecuencia e intensidad de dolor (ambos $\mathrm{p}<0,05)$, pero no tuvo efecto en el test de hidrógeno, producción de metano, características de la heces o tiempo de tránsito ${ }^{41}$. Un meta análisis reciente ${ }^{42}$ concluyó, al aplicar la gradación GRADE, que la evidencia disponible en SII resulta de muy baja calidad y no respalda la recomendación de una dieta baja en FODMAPs ni la DLG.

\section{Problemas diagnósticos}

No existe metodología validada para hacer diagnóstico. Hasta ahora existen dos tipos de aproximación, el test de hidrógeno espirado con agentes específicos, que permiten diagnosticar intolerancia a algunos carbohidratos (lactosa, sacarosa, sorbitol ${ }^{43}$ o fructosa ${ }^{44}$ ) y pruebas de desafío, en general basadas en procedimientos para estudiar alergias, pero aún se discute la manera más apropiada de realizarlas. Dada la variedad de FODMAPs que existen, el enorme número de alimentos y productos procesados que los contienen y la variabilidad que presentan las personas afectadas, las pruebas utilizadas son numerosas, variables, y aúnan el proceso diagnóstico con la implementación del tratamiento.

\section{La dieta baja en FODMAPs}

Existe evidencia que indica que la dieta baja en FODMAPs puede ser beneficiosa tanto en niños como adultos, por lo que, mientras no haya información más definitiva, se le plantea como terapia. Está ensayada principalmente en pacientes con SII, es difícil de realizar porque implica restringir drásticamente el consumo de los muchos FODMAPs de la dieta ${ }^{45}$ (Tabla 2). Una frecuentemente usada, describe 3 fases ${ }^{46}$. En la evaluación inicial se deben detallar los síntomas asociados, dar asesoramiento médico-nutricional, explicar al paciente la dieta, que no es fácil de realizar, sus efectos y que es altamente restrictiva. Es fundamental seguir la secuencia de manera estricta. La primera fase consiste en eliminar todas las fuentes de FODMAPs, para llevar su concentración por debajo del umbral que genera síntomas. La duración de esta fase se describe entre 3-8 semanas ${ }^{45,47}$; en la práctica, 3-4 semanas son generalmente suficientes para lograr mejoría de los síntomas, pero algunos pacientes requieren más tiempo. Si durante esta etapa no hay respuesta satisfactoria, y la dieta es bien realizada, se debe reintroducir la dieta habitual y considerar otros diagnósticos diferenciales. Si se logra disminución de síntomas, se inicia la segunda fase, que reintroduce alimentos de manera controlada y por etapas, para identificar el umbral de aparición de síntomas de cada compuesto. Una forma frecuente de reintroducción consiste en incorporar un alimento alto en alguno de los tipos de FODMAPs por semana, administrándolo por 3 días en dosis crecientes, durante los cuales se registra la presencia de síntomas, y los 4 días restantes de la semana se vuelve a la dieta inicial, sin FODMAPs (Figura 2). Por ejemplo, para reintroducir lactosa, se indica consumir $1 / 2$ taza de leche el lunes, 1 taza el martes y $1 \frac{1}{2} 2$ el miércoles; si el paciente presenta síntomas el lunes o martes se detiene la prueba y se cataloga como compuesto no tolerado; si no aparecen síntomas, la prueba finaliza el miércoles y de jueves a domingo se vuelve a la dieta baja en FODMAPs inicial. A la semana siguiente se prueba con un alimento diferente usando el mismo esquema, y así hasta evaluar todos los tipos de FODMAPs. Esta etapa debería durar 6-10 semanas ${ }^{48}$. La última fase corresponde al ajuste (personalización) de la dieta, reintroduciendo los alimentos que no se asociaron a síntomas en la etapa previa y en cantidades que se establecieron como no generadoras de síntomas.

\section{Dificultades de la dieta}

Por ser restrictiva, una de las complicaciones de la dieta baja en FODMAPs es el potencial compromiso nutricional. Puede disminuir el peso debido a la menor ingesta de energía ${ }^{49}$. Si hay bajo consumo de lácteos se debe monitorear la ingesta de calcio y la salud ósea ${ }^{50}$, aunque algunos estudios han mostrado que los niveles de calcio pueden mantenerse con ingesta de lácteos sin lactosa ${ }^{49}$. Los datos acerca de la ingesta de fibra son controversiales. Hay ensayos que muestran que la ingesta de fibra disminuye ${ }^{51}$, por restricción de cereales y legumbres, pero otros muestran que el consumo no cambia por el consumo de fibra baja en FODMAPs $^{39}$. Puede haber disminución del contenido de agua intestinal ${ }^{52}$, que favorece la aparición o exacerbación de constipación, pero otros estudios muestran mejoría de los síntomas, probablemente 


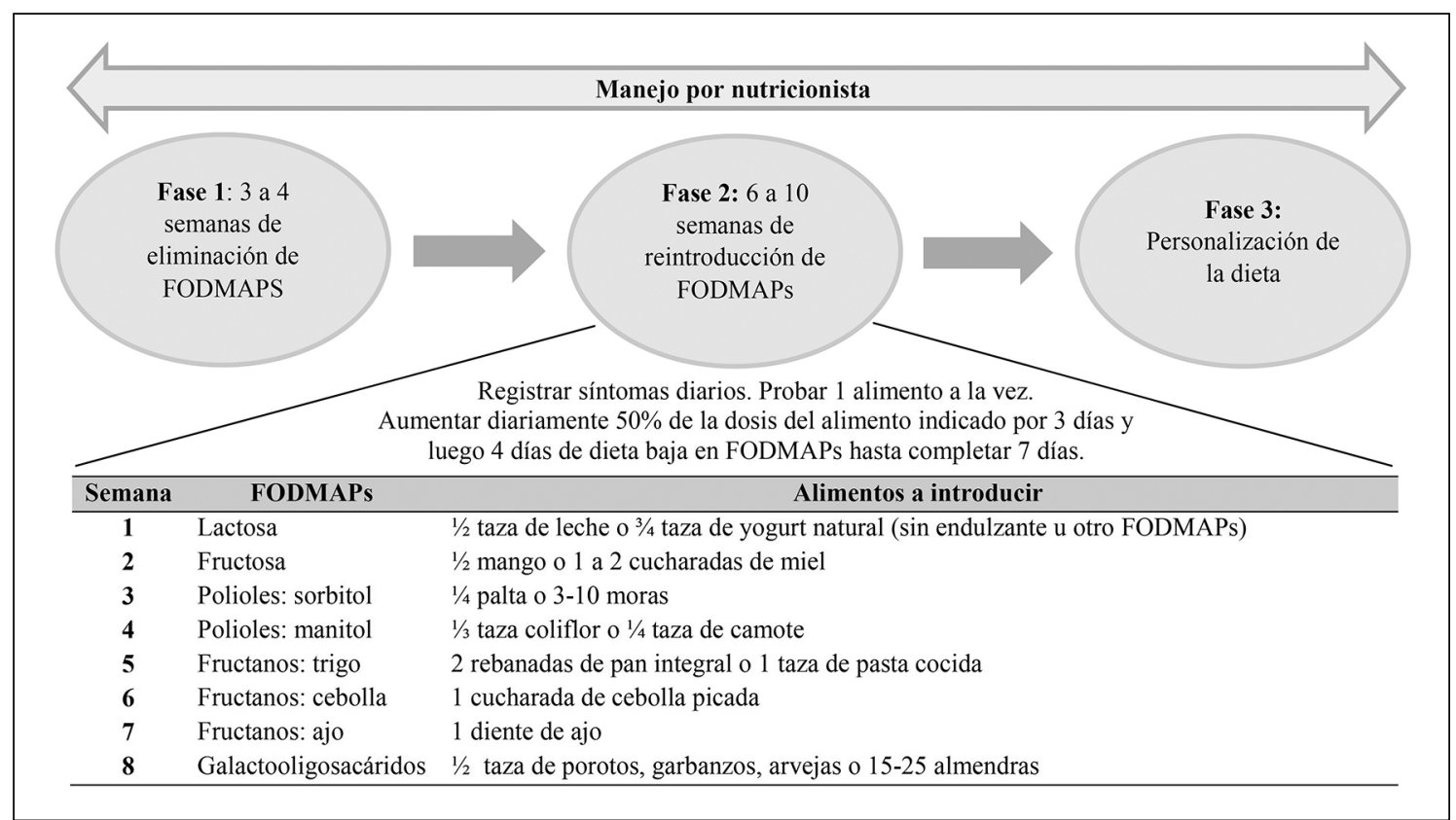

Figura 2. Plan en tres fases para realizar una dieta baja en FODMAPs.

porque la dieta es más equilibrada ${ }^{53}$. Al igual que en otras dietas restrictivas, puede haber disminución de micronutrientes y vitaminas del grupo B, antioxidantes naturales como flavonoides, carotenoides y vitamina $\mathrm{D}^{54}$.

Sin duda el rol de un profesional especializado en este tema es crucial para el manejo exitoso a largo plazo del paciente. Se recomienda que el consejo nutricional sea guiado por un/a nutricionista ${ }^{55}$, que oriente al paciente en identificar alimentos altos y bajos FODMAPs y entregue información práctica acerca del etiquetado nutricional de los productos procesados, ya que estos no entregan información de su contenido de FODMAPs. Ya en tratamiento, es necesario evaluar y promover la adherencia a la dieta. Esta, es de mayor costo que la habitual, dificulta la relación del paciente con quienes comparte la vida diaria y puede afectar sus relaciones sociales y laborales.

En resumen, históricamente era conocido que el consumo de alimentos ricos en FODMAPs puede asociarse, ocasionalmente, a manifestaciones clínicas. La dieta occidental de hoy día, en cambio, es alta en FODMAPs y produce síntomas que afectan cada día a un mayor segmento de la población. Poner al día los conocimientos en el tema, generar métodos diagnósticos y tratamientos reproducibles y confiables, y promover el consumo razonable de FODMAPs es tarea de todos los profesionales de la salud.

\section{Referencias}

1. Gibson PR. History of the low FODMAP diet. J Gastroenterol Hepatol 2017; 32 (Suppl 1): 5-7.

2. McMichael HB, Webb J, Dawson AM. Lactase deficiency in adults a cause of "functional" diarrhoea. Lancet 1965; 285 (7388): 717-20.

3. Scheinin A, Mäkinen KK. Turku sugar studies: An overview. Acta Odontol Scand 1976; 34 (6): 405-8.

4. Andersson DE, Nygren A. Four cases of long-standing diarrhoea and colic pains cured by fructose-free diet. A pathogenetic discussion. Acta Med Scand 1978; 203 (1-2): 87-92.

5. Stone-Dorshow T, Levitt MD. Gaseous response to ingestion of a poorly absorbed fructo-oligosaccharide sweetener. Am J Clin Nutr 1987; 46 (1): 61-5.

6. Gibson PR, Shepherd SJ. Personal view: food for thought-western lifestyle and susceptibility to Crohn's disease. The FODMAP hypothesis. Aliment Pharmacol Ther 2005; 21 (12): 1399-409. 
7. Jones HF, Butler RN, Brooks DA. Intestinal fructose transport and malabsorption in humans. Am J Physiol Gastrointest Liver Physiol 2011; 300 (2): G202-6.

8. Deng Y, Misselwitz B, Dai N, Fox M. Lactose intolerance in adults: biological mechanism and dietary management. Nutrients 2015; 7 (9): 8020-35.

9. Roberfroid M, Delzenne NM. Dietary fructans. Annu Rev Nutr 1998; 18 (1): 117-43.

10. Tuck CJ, Muir JG, Barrett JS, Gibson PR. Fermentable oligosaccharides, disaccharides, monosaccharides and polyols: role in irritable bowel syndrome. Expert Rev Gastroenterol Hepatol 2014; 8 (7): 819-34.

11. Yao C, Tan HL, van Langenberg DR, Barrett JS, Rose R, Liels K, et al. Dietary sorbitol and mannitol: food content and distinct absorption patterns between healthy individuals and patients with irritable bowel syndrome. J Hum Nutr Diet 2013; 27 (Suppl. 2): 263-75.

12. Spiller R. How do FODMAPs work? J Gastroenterol Hepatol 2017; 32 (Suppl 1): 36-9.

13. Marciani L, Cox EF, Hoad CL, Pritchard S, Totman JJ, Foley S, et al. Postprandial changes in small bowel water content in healthy subjects and patients with irritable bowel syndrome. Gastroenterology 2010; 138 (2): 46977.e1.

14. Murray K, Wilkinson-Smith V, Hoad C, Costigan C, Cox E, Lam C, et al. Differential effects of FODMAPs (fermentable oligo-, di-, mono-saccharides and polyols) on small and large intestinal contents in healthy subjects shown by MRI. Am J Gastroenterol 2014; 109 (1): 110-9.

15. Major G, Pritchard S, Murray K, Alappadan JP, Hoad $\mathrm{CL}$, Marciani L, et al. Colon hypersensitivity to distension, rather than excessive gas production, produces carbohydrate-related symptoms in individuals with irritable bowel syndrome. Gastroenterology 2017; 152 (1): 124-33.e2.

16. Muir JG, Shepherd SJ, Rosella O, Rose R, Barrett JS, Gibson PR. Fructan and free fructose content of common Australian vegetables and fruit. J Agric Food Chem 2007; 55 (16): 6619-27.

17. Muir JG, Rose R, Rosella O, Liels K, Barrett JS, Shepherd $\mathrm{SJ}$, et al. Measurement of short-chain carbohydrates in common Australian vegetables and fruits by high-performance liquid chromatography (HPLC). J Agric Food Chem 2009; 57 (2): 554-65.

18. Biesiekierski JR, Rosella O, Rose R, Liels K, Barrett JS, Shepherd SJ, et al. Quantification of fructans, galacto-oligosacharides and other short-chain carbohydrates in processed grains and cereals. J Hum Nutr Diet 2011; 24 (2): 154-76.

19. Varney J, Barrett J, Scarlata K, Catsos P, Gibson PR, Muir JG. FODMAPs: food composition, defining cutoff values and international application. J Gastroenterol Hepatol 2017; 32 (Suppl 1): 53-61.

20. Halmos EP, Power VA, Shepherd SJ, Gibson PR, Muir JG. A diet low in FODMAPs reduces symptoms of irritable bowel syndrome. Gastroenterology 2014; 146 (1): 67-75.e5.

21. Barrett JS, Gibson PR. Fermentable oligosaccharides, disaccharides, monosaccharides and polyols (FODMAPs) and nonallergic food intolerance: FODMAPs or food chemicals? Ther Adv Gastroenterol 2012; 5 (4), 261-8.

22. Holscher HD. Dietary fiber and prebiotics and the gastrointestinal microbiota. Gut Microbes 2017; 8 (2): 172-84.

23. Ahmadi S, Mainali R, Nagpal R, Sheikh-Zeinoddin M, Soleimanian-Zad S, Wang S, et al. Dietary polysaccharides in the amelioration of gut microbiome dysbiosis and metabolic diseases. Obes Control Ther 2017; 4 (3): 1-15.

24. He M, Shi B. Gut microbiota as a potential target of metabolic syndrome: the role of probiotics and prebiotics. Cell Biosci 2017; 7: 54.

25. Li G, Xie C, Lu S, Nichols RG, Tian Y, Li L, et al. Intermittent fasting promotes white adipose browning and decreases obesity by shaping the gut microbiota. Cell Metab 2017; 26 (4): 672-85.e4.

26. Matthews SB, Waud JP, Roberts AG, Campbell AK. Systemic lactose intolerance: a new perspective on an old problem. Postgrad Med J 2005; 81 (953): 167-73.

27. Campbell AK, Matthews SB, Vassel N, Cox CD, Naseem $\mathrm{R}$, Chaichi J, et al. Bacterial metabolic 'toxins': a new mechanism for lactose and food intolerance, and irritable bowel syndrome. Toxicology 2010; 278 (3): 268-76.

28. Würsch P, Koellreutter B, Schweizer TF. Hydrogen excretion after ingestion of five different sugar alcohols and lactulose. Eur J Clin Nutr 1989; 43 (12): 819-25.

29. Halmos EP, Christophersen CT, Bird AR, Shepherd SJ, Gibson PR, Muir JG. Diets that differ in their FODMAP content alter the colonic luminal microenvironment. Gut 2015; 64 (1): 93-100.

30. Sartin JS. Gastrointestinal disorders. En: Copstead LC, Banasik JL, Editores, Pathophysiology. Philadelphia: Elsevier Inc.; 2005. p. 887-911.

31. Jenkins DJ, Reynolds R, Slavin B, Leeds AR, Jenkins AL, Jepson EM. Dietary fiber and blood lipids: treatment of hypercholesterolemia with guar gum crispbread. Am J Clin Nutr 1980; 33 (3): 575-81.

32. Livesey G. Tolerance of low-digestible carbohydrates: a general view. Brit J Nutr 2001; 85 (Suppl 1): S7-16.

33. Nyman M. Fermentation and bulking capacity of indigestible carbohydrates: the case of inulin and oligofructose. Brit J Nutr 2002; 87 (Suppl 2): S163-8.

34. Stephen A. Starch and dietary fiber: their physiological 
and epidemiological interrelationships. Can J Phys Pharmacol 1991; 69 (1): 116-20.

35. Cummings JH, Macfarlane GT. Gastrointestinal effects of prebiotics. Brit J Nutr 2002. 87 (Suppl 2): S145-51.

36. Biesiekierski JR, Newnham ED, Irving PM, Barrett JS, Haines M, Doecke JD, et al. Gluten causes of gastrointestinal symptoms in subjects without celiac disease: a double-blind randomized placebo controlled trial. Am J Gastroenterol. 2011; 106 (3): 508-14.

37. Biesiekierski JR, Peters SL, Newnham ED, Rosella O, Muir JG, Gibson PR. No effects of gluten in patients with self-reported non-celiac gluten sensitivity after dietary reduction of fermentable, poorly absorbed shortchain carbohydrates. Gastroenterology 2013; 145 (2): 320-8.

38. Shahbazkhani B, Sadeghi A, Malekzadeh R, Khatavi F, Etemadi M, Kalantri E, et al. Non-celiac gluten sensitivity has narrowed the spectrum of irritable bowel syndrome: a double-blind randomized placebo-controlled trial. Nutrients 2015; 7(6): 4542-54.

39. Eswaran SL, Chey WD, Han-Markey T, Ball S, Jackson $\mathrm{K}$. A randomized controlled trial comparing the low FODMAP diet vs. modified NICE guidelines in US adults with IBS-D. Am J Gastroenterol 2016; 111 (12): 1824-32.

40. Böhn L, Störsrud S, Liljebo T, Collin L, Lindfors P, Törnblom $\mathrm{H}$, et al. Diet low in FODMAPs reduces symptoms of irritable bowel syndrome as well as traditional dietary advice: a randomized controlled trial, Gastroenterology 2015; 149 (6): 1399-407.e2.

41. Chumpitazi BP, Hollister EB, Oezguen N, Tsai CM, McMeans AR, Luna RA, et al. Gut microbiota influences low fermentable substrate diet efficacy in children with irritable bowel syndrome. Gut Microbes 2014; 5 (2): 165-75.

42. Dionne J, Ford AC, Yuan Y, Chey WD, Lacy BE, Saito YA, et al. A systematic review and meta-analysis evaluating the efficacy of a gluten-free diet and a low FODMAPs diet in treating symptoms of irritable bowel syndrome. Am J Gastroenterol 2018; 113 (9): 1290-300.

43. Berni Canani R, Pezzella V, Amoroso A, Cozzolino T, Di Scala C, Passariello A. Diagnosing and treating intolerance to carbohydrates in children. Nutrients 2016; 8 (3): 157.

44. Montalto M, Gallo A, Ojetti V, Gasbarrini A. Fructose, trehalose and sorbitol malabsorption. Eur Rev Med Pharmacol Sci 2013; 17 (Suppl 2): 26-9.

45. Staudacher HM, Whelan K. The low FODMAP diet: recent advances in understanding its mechanisms and efficacy in irritable bowel syndrome. Gut 2017; 66 (8): 1517-27.
46. Whelan K, Martin LD, Staudacher HM, Lomer MCE. The low FODMAP diet in the management of irritable bowel syndrome: an evidence-based review of FODMAP restriction, reintroduction and personalisation in clinical practice. J Hum Nutr Diet 2018; 31 (2): 239-55.

47. Hustoft TN, Hausken T, Ystad SO, Valeur J, Brokstad K, Hatlebakk JG, et al. Effects of varying dietary content of fermentable short-chain carbohydrates on symptoms, fecal microenvironment, and cytokine profiles in patients with irritable bowel syndrome. Neurogastroenterol Motil 2017; 29 (4): e12969.

48. Vakil N. Dietary fermentable oligosaccharides, disaccharides, monosaccharides, and polyols (FODMAPs) and gastrointestinal disease. Nutr Clin Pract 2018; 33 (4): 468-75.

49. O’Keeffe M, Jansen C, Martin L, Williams M, Seamark L, Staudacher HM, et al. Long-term impact of the low-FODMAP diet on gastrointestinal symptoms, dietary intake, patient acceptability, and healthcare utilization in irritable bowel syndrome. Neurogastroenterol Motil 2018; 30 (1): e13154.

50. Staudacher HM, Lomer MC, Anderson JL, Barrett JS, Muir JG, Irving PM, et al. Fermentable carbohydrate restriction reduces luminal bifidobacteria and gastrointestinal symptoms in patients with irritable bowel syndrome. J Nutr 2012; 142 (8): 1510-8.

51. Böhn L, Störsrud S, Liljebo T, Collin L, Lindfors P, Törnblom H, et al. Diet low in FODMAPs reduces symptoms of irritable bowel syndrome as well as traditional dietary advice: a randomized controlled trial. Gastroenterology 2015; 149 (6): 1399-407.e2.

52. Barrett JS, Gearry RB, Muir JG, Irving PM, Rose R, Rosella O, et al. Dietary poorly absorbed, short-chain carbohydrates increase delivery of water and fermentable substrates to the proximal colon. Aliment Pharmacol Ther 2010; 31 (8): 874-82.

53. de Roest RH, Dobbs BR, Chapman BA, Batman B, O'Brien LA, Leeper JA, et al. The low FODMAP diet improves gastrointestinal symptoms in patients with irritable bowel syndrome: a prospective study. Int J Clin Pract 2013; 67 (9): 895-903.

54. Gupta S, Schaffer G, Saps M. Pediatric irritable bowel syndrome and other functional abdominal pain disorders: an update of non-pharmacological treatments. Expert Rev Gastroenterol Hepatol 2018; 12 (5): 447-56.

55. Whigham L, Joyce T, Harper G, Irving PM, Staudacher $\mathrm{HM}$, Whelan $\mathrm{K}$, et al. Clinical effectiveness and economic costs of group versus one- to-one education for short-chain fermentable carbohydrate restriction (low FODMAP diet) in the management of irritable bowel syndrome. J Hum Nutr Diet 2015; 28 (6): 687-96. 